\title{
Uniqueness of the potential function for the vectorial Sturm-Liouville equation on a finite interval
}

Tsorng-Hwa Chang ${ }^{1,2}$ and Chung-Tsun Shieh ${ }^{1 *}$

\author{
* Correspondence: ctshieh@mail. \\ tku.edu.tw \\ 'Department of Mathematics, \\ Tamkang University, No.151, \\ Yingzhuan Rd., Danshui Dist., New \\ Taipei City 25137, Taiwan, PR China \\ Full list of author information is \\ available at the end of the article
}

\begin{abstract}
In this paper, the vectorial Sturm-Liouville operator $L_{Q}=-\frac{\mathrm{d}^{2}}{\mathrm{~d} x^{2}}+Q(x)$ is considered, where $Q(x)$ is an integrable $m \times m$ matrix-valued function defined on the interval $[0, \pi]$ The authors prove that $m^{2}+1$ characteristic functions can determine the potential function of a vectorial Sturm-Liouville operator uniquely. In particular, if $Q(x)$ is real symmetric, then $\frac{m(m+1)}{2}+1$ characteristic functions can determine the potential function uniquely. Moreover, if only the spectral data of self-adjoint problems are considered, then $m^{2}+1$ spectral data can determine $Q(x)$ uniquely.
\end{abstract}

Keywords: Inverse spectral problems, Sturm-Liouville equation

\section{Introduction}

The study on inverse spectral problems for the vectorial Sturm-Liouville differential equation

$$
\vec{y}^{\prime \prime}+\left(\lambda I_{m}-Q(x)\right) \vec{y}=0, \quad 0<x<\pi,
$$

on a finite interval is devoted to determine the potential matrix $Q(x)$ from the spectral data of (1.1) with boundary conditions

$$
U(\vec{\gamma}):=\vec{\gamma}^{\prime}(0)-h \vec{\gamma}(0)=0, \quad V(\vec{\gamma}):=\vec{\gamma}^{\prime}(\pi)+H \vec{\gamma}(\pi)=0,
$$

where $\lambda$ is the spectral parameter, $h=\left[h_{i j}\right]_{i, j=\overline{1, m}}$ and $H=\left[H_{i j}\right]_{i, j=1, m}$ are in $M_{n}(\mathbb{C})$ and $Q(x)=\left[Q_{i j}(x)\right]_{i, j=\overline{1, m}}$ is an integrable matrix-valued function. We use $L_{m}=L(Q, h, H)$ to denote the boundary problem (1.1)-(1.2). For the case $m=1,(1.1)-(1.2)$ is a scalar Sturm-Liouville equation. The scalar Sturm-Liouville equation often arises from some physical problems, for example, vibration of a string, quantum mechanics and geophysics. Numerous research results for this case have been established by renowned mathematicians, notably Borg, Gelfand, Hochstadt, Krein, Levinson, Levitan, Marchenko, Gesztesy, Simon and their coauthors and followers (see [1-9] and references therein). For the case $m \geq 2$, some interesting results had been obtained (see [10-20]). In particular, for $m=2$ and $Q(x)$ is a two-by-two real symmetric matrix-valued smooth functions defined in the interval $[0, \pi]$ Shen [18] showed that five spectral data can 
determine $Q(x)$ uniquely. More precisely speaking, he considered the inverse spectral problems of the vectorial Sturm-Liouville equation:

$$
\vec{\gamma}^{\prime \prime}+\left(\lambda I_{2}-Q_{2}(x)\right) \vec{\gamma}(x)=0, \quad 0<x<\pi,
$$

where $Q_{2}(x)$ is a real symmetric matrix-valued function defined in the interval $[0, \pi]$. Let $\sigma_{D}(Q)$ denotes the Dirichlet spectrum of (1.3), $\sigma_{N D}(Q)$ the Neumann-Dirichlet spectrum of (1.3) and $\sigma_{j}(Q)$ the spectrum of (1.3) with boundary condition

$$
\vec{\gamma}^{\prime}(0)-B_{j} \vec{\gamma}(0)=\vec{\gamma}(\pi)=\overrightarrow{0},
$$

for $j=1,2,3$, where

$$
B_{j}=\left[\begin{array}{ll}
\alpha_{j} & \beta_{j} \\
\beta_{j} & \gamma_{j}
\end{array}\right]
$$

is a real symmetric matrix and $\left\{\left(\alpha_{j}, \beta_{j}, \gamma_{j}\right), j=1,2,3\right\}$ is linearly independent over $\mathbb{R}$. Then

Theorem 1.1 ([18], Theorem 4.1). Let $Q_{2}(x)$ and $\widetilde{Q}_{2}(x)$ be two continuous two-by-two real symmetric matrix-valued functions defined on $[0, \pi]$. Suppose that $\sigma_{N D}(\widetilde{Q})=\sigma_{N D}(\widetilde{Q}) \sigma_{N D}(\widetilde{Q})=\sigma_{N D}(\widetilde{Q})$ and $\sigma_{j}(Q)=\sigma_{j}(\widetilde{Q})$ for $j=1,2,3$, then $Q(x)=\widetilde{Q}(x)$ on $[0, \pi]$.

The purpose of this paper is to generalize the above theorem for the case $m \geq 3$. The idea we use is the Weyl's matrix for matrix-valued Sturm-Liouville equation

$$
Y^{\prime \prime}+\left(\lambda I_{m}-Q(x)\right) Y=0, \quad 0<x<\pi .
$$

Some uniqueness theorems for vectorial Sturm-Liouville equation are obtained in the last section.

\section{Main Results}

Let $C(x, \lambda)=\left[C_{i j}(x, \lambda)\right]_{i, j=\overline{1, m}}$ and $S(x, \lambda)=\left[S_{i j}(x, \lambda)\right]_{i, j=\overline{1, m}}$ be two solutions of equation (1.5) which satisfy the initial conditions

$$
\begin{aligned}
& C(0, \lambda)=S^{\prime}(0, \lambda)=I_{m} \\
& C^{\prime}(0, \lambda)=S(0, \lambda)=0_{m}
\end{aligned}
$$

where $0_{m}$ is the $m \times m$ zero matrix, $I_{m}=\left[\delta_{i j}\right]_{i, j=1, m}$ is the $m \times m$ identity matrix and $\delta_{i j}$ is the Kronecker symbol. For given complex-valued matrices $h$ and $H$, we denote

$$
\varphi(x, \lambda)=\left[\varphi_{i j}(x, \lambda)\right]_{i, j=\overline{1, m}} \text { and } \Phi(x, \lambda)=\left[\Phi_{i j}(x, \lambda)\right]_{i, j=\overline{1, m}}
$$

be two solutions of equation (1.5) so that $\phi(x, \lambda)=C(x, \lambda)+S(x, \lambda) h$ and $\Phi(x, \lambda)=S(x, \lambda)+\varphi(x, \lambda) \mathcal{M}(\lambda)$ which satisfy the boundary conditions

$$
\left\{\begin{array}{l}
U(\Phi)=\Phi^{\prime}(0)-h \Phi(0)=I_{m} \\
V(\Phi)=\Phi^{\prime}(\pi)+H \Phi(\pi)=0_{m} .
\end{array}\right.
$$

Then, $\mathcal{M}(\lambda)=\Phi(0, \lambda)$. The matrix $\mathcal{M}(\lambda)=\left[\mathcal{M}_{i j}(\lambda)\right]_{i, j=1, m}$ is called the Weyl matrix for $L_{m}(Q, h, H)$. In 2006, Yurko proved that:

Theorem 2.1 ([20], Theorem 1). Let $\mathcal{M}(\lambda)$ and $\widetilde{\mathcal{M}}(\lambda)$ denote Weyl matrices of the problems $L_{m}(Q, h, H)$ and $L_{m}(\widetilde{Q}, \tilde{h}, \tilde{H})$ separately. Suppose $\mathcal{M}(\lambda)=\widetilde{\mathcal{M}}(\lambda)$, then $h=\tilde{h}, h=\tilde{h}$ and $H=\tilde{H}$. 
Also note that from [20], we have

$$
\begin{aligned}
& \Phi(x, \lambda)=S(x, \lambda)+\varphi(x, \lambda) \mathcal{M}(\lambda)=\psi(x, \lambda)(U(\psi))^{-1}, \\
& \mathcal{M}(\lambda)=-(V(\varphi))^{-1} V(S)=\psi(0, \lambda)(U(\psi))^{-1}
\end{aligned}
$$

where $\psi(x, \lambda)=\left[\psi_{i j}(x, \lambda)\right]_{i, j=1, m}$ is a matrix solution of equation (1.5) associated with the conditions $\psi(\pi, \lambda)=I_{m}$ and $\psi^{\prime}(\pi, \lambda)=-H$. It is not difficult to see that both $\Phi(x, \lambda)$ and $\mathcal{M}(\lambda)$ are meromorphic in $\lambda$ and the poles of $\mathcal{M}(\lambda)$ are coincided with the eigenvalues of $L_{m}(Q, h, H)$. Moreover, we have

$$
\mathcal{M}(\lambda)=-(V(\varphi))^{-1} V(S)=-\frac{\operatorname{Adj}\left(\varphi^{\prime}(\pi, \lambda)+H \varphi(\pi, \lambda)\right)}{\operatorname{det}\left(\varphi^{\prime}(\pi, \lambda)+H \varphi(\pi, \lambda)\right)}\left(S^{\prime}(\pi, \lambda)+H S(\pi, \lambda)\right),
$$

where $\operatorname{Adj}(A)$ denotes the adjoint matrix of $A$ and $\operatorname{det}(A)$ denotes the determinant of $A$. In the remaining of this section, we shall prove some uniqueness theorems for vectorial Sturm-Liouville equations. Let $B(i, j)=\left[b_{r s}\right]_{r, s=\overline{1, m}}$,

$$
b_{r s}=\left(\begin{array}{ll}
0, & (r, s) \neq(i, j), \quad 1 \leq i, j \leq m, \\
1, & (r, s)=(i, j),
\end{array}\right.
$$

and $B(0,0)=0_{m}$ The characteristic function for this boundary value problem $L_{m}(Q, h+$ $B(i, j), H)$ is

$$
\Delta_{i j}(\lambda)=\operatorname{det}(V(\varphi+S B(i, j))), \quad 1 \leq i, j \leq m \text { or }(i, j)=(0,0) .
$$

The first problem we want to study is as following:

Problem 1. How many $\Delta_{i j}(\lambda)$ can uniquely determine $Q, h$ and $H$ ? where $(i, j)=(0$,

0) or $1 \leq i, j \leq m$

To find the solution of Problem 1, we start with the following lemma

Lemma 2.2. Let $B(i, j)=\left[b_{r s}\right]_{m \times m}$ and $\Delta_{i j}$ be defined as above. Then

$$
\begin{aligned}
\Delta_{i j}(\lambda)= & \Delta_{00}(\lambda)+\operatorname{det}\left(\text { Augment } \left[\varphi_{1}^{\prime}(\pi, \lambda)+H \varphi_{1}(\pi, \lambda), \ldots,\right.\right. \\
& \left.\left.S_{i}^{\prime}(\pi, \lambda)+H S_{i}(\pi, \lambda), \ldots, \varphi_{m}^{\prime}(\pi, \lambda)+H \varphi_{m}(\pi, \lambda)\right]\right),
\end{aligned}
$$

where $\phi_{k}(\pi, \lambda)$ is the kth column of $\phi(\pi, \lambda)$ and $S_{k}(\pi, \lambda)$ the kth column of $S(\pi, \lambda)$ for $k=1,2,3, \ldots, m$.

Proof. Let

$$
\begin{aligned}
Y(x, \lambda) & =[C(x, \lambda)+S(x, \lambda)(h+B(i, j))] \\
& =[(C(x, \lambda)+S(x, \lambda) h)+S(x, \lambda) B(i, j)] \\
& =[\varphi(x, \lambda)+S(x, \lambda) B(i, j)]
\end{aligned}
$$

Then

$$
\begin{aligned}
\Delta_{i j}(\lambda)= & \operatorname{det}\left(Y^{\prime}(\pi, \lambda)+H Y(\pi, \lambda)\right) \\
= & \operatorname{det}\left(\left(\varphi^{\prime}(\pi, \lambda)+H \varphi(\pi, \lambda)\right)+\left(S^{\prime}(\pi, \lambda)+H S(\pi, \lambda) B(i, j)\right)\right. \\
= & \operatorname{det}\left(\left(\varphi^{\prime}(\pi, \lambda)+H \varphi(\pi, \lambda)\right)+\left[0, S_{i}^{\prime}(\pi, \lambda)+H S_{i}(\pi, \lambda) 0\right]\right) \\
= & \operatorname{det}\left(\varphi^{\prime}(\pi, \lambda)+H \varphi(\pi, \lambda)\right)+\operatorname{det}\left(\varphi_{1}^{\prime}(\pi, \lambda)+H \varphi_{1}(\pi, \lambda), \ldots,\right. \\
& \quad(\text { jth column }) \\
& \left.S_{i}^{\prime}(\pi, \lambda)+H S_{i}(\pi, \lambda), \ldots, \varphi_{m}^{\prime}(\pi, \lambda)+H \varphi_{m}(\pi, \lambda)\right) \\
= & \Delta_{00}(\lambda)+\operatorname{det}\left(\varphi_{m}^{\prime}(\pi, \lambda)+H \varphi_{1}(\pi, \lambda), \ldots,\right. \\
& \quad \quad(\text { th column }) \\
& \left.S_{i}^{\prime}(\pi, \lambda)+H S_{i}(\pi, \lambda), \ldots, \varphi_{m}^{\prime}(\pi, \lambda)+H \varphi_{m}(\pi, \lambda)\right) .
\end{aligned}
$$


Next, we shall prove the first main theorem. For simplicity, if a symbol $\alpha$ denotes an object related to $L_{m}(Q, h, H)$, then the symbol $\tilde{\alpha}$ denotes the analogous object related to $L_{m}(\widetilde{Q}, \tilde{h}, \widetilde{H})$.

Theorem 2.3. Suppose that $\Delta_{i j}(\lambda)=\widetilde{\Delta}_{i j}(\lambda)$ for $(i, j)=(0,0)$ or $1 \leq i, j \leq m$ then $h=\tilde{h}, h=\tilde{h}$ and $H=\tilde{H}$.

Proof. Since

$$
0_{m}=\Phi^{\prime}(\pi, \lambda)+H \Phi(\pi, \lambda)
$$

and

$$
\Phi(x, \lambda)=S(x, \lambda)+\varphi(x, \lambda) \mathcal{M}(\lambda)
$$

we have that

$$
-\left(S^{\prime}(\pi, \lambda)+H S(\pi, \lambda)\right) e_{i}=\left(\varphi^{\prime}(\pi, \lambda)+H \varphi(\pi, \lambda)\right) \mathcal{M}(\lambda) e_{i}
$$

for each $i=1, \ldots, m$, that is,

$$
-\left(S_{i}^{\prime}(\pi, \lambda)+H S_{i}(\pi, \lambda)\right)=\left(\varphi^{\prime}(\pi, \lambda)+H \varphi(\pi, \lambda)\right) \mathcal{M}_{i}(\lambda) .
$$

By Crammer's rule,

$$
\begin{aligned}
& \mathcal{M}_{j i}(\lambda) \\
= & \frac{-\operatorname{det}\left(\varphi_{1}^{\prime}(\pi, \lambda)+H \varphi_{1}(\pi, \lambda), \ldots, S_{i}^{\prime}(\pi, \lambda)+H S_{i}(\pi, \lambda), \ldots, \varphi_{m}^{\prime}(\pi, \lambda)+H \varphi_{m}(\pi, \lambda)\right)}{\operatorname{det}\left(\varphi^{\prime}(\pi, \lambda)+H \varphi(\pi, \lambda)\right)} \\
= & \frac{\Delta_{00}(\lambda)-\Delta_{i j}(\lambda)}{\Delta_{00}(\lambda)} \\
= & \frac{\widetilde{\Delta}_{00}(\lambda)-\widetilde{\Delta}_{i j}(\lambda)}{\widetilde{\Delta}_{00}(\lambda)} \\
= & \widetilde{\mathcal{M}}_{j i}(\lambda) \text { for } 1 \leq i, j \leq m .
\end{aligned}
$$

Applying Theorem 2.1, we conclude that $Q=\widetilde{Q}, h=\tilde{h}$ and $H=\widetilde{H}$.

Lemma 2.4. Suppose that $h, H$ are real symmetric matrices and $Q(x)$ is a real symmetric matrix-valued function. Then, $\mathcal{M}(\lambda)=-V(\varphi)^{-1} V(S)$ is real symmetric for all $\lambda$ $\in \mathbb{R}$.

Proof. Let

$$
U(x, \lambda)=\left[\begin{array}{cc}
\varphi^{\prime}(x, \lambda) & S^{\prime}(x, \lambda) \\
\varphi(x, \lambda) & S(x, \lambda)
\end{array}\right]
$$

For $\lambda \in \mathbb{R}$,

$$
\left\{\begin{array}{l}
\left(S^{\prime *} \varphi-S^{*} \varphi^{\prime}\right)(x, \lambda)=\left(S^{* *} \varphi-S^{*} \varphi^{\prime}\right)(0, \lambda)=I_{m} \\
\left(S^{\prime *} S-S^{*} S^{\prime}\right)(x, \lambda)=\left(S^{\prime *} S-S^{*} S^{\prime}\right)(0, \lambda)=0_{m} \\
\left(\varphi^{*} \varphi^{\prime}-\varphi^{\prime *} \varphi\right)(x, \lambda)=\left(\varphi^{*} \varphi^{\prime}-\varphi^{\prime *} \varphi\right)(0, \lambda)=0_{m} \\
\left(\varphi^{*} S^{\prime}-\varphi^{\prime *} S\right)(x, \lambda)=\left(\varphi^{*} S^{\prime}-\varphi^{\prime *} S\right)(0, \lambda)=I_{m}
\end{array}\right.
$$

This leads to

$$
U^{-1}(x, \lambda)=\left[\begin{array}{cc}
-(S)^{*}(x, \lambda) & \left(S^{*}\right)^{\prime}(x, \lambda) \\
\varphi^{*}(x, \lambda) & -\left(\varphi^{*}\right)^{\prime}(x, \lambda)
\end{array}\right] .
$$


Now let

$$
U_{2}(x, \lambda)=\left[\begin{array}{cc}
I_{m} & H \\
0 & I_{m}
\end{array}\right] U(x, \lambda) .
$$

Then

$$
U_{2}(1, \lambda)=\left[\begin{array}{cc}
I_{m} & H \\
0 & I_{m}
\end{array}\right] U(1, \lambda)=\left[\begin{array}{cc}
V(\varphi) & V(S) \\
\varphi(1, \lambda) & S(1, \lambda)
\end{array}\right]
$$

and

$$
U_{2}^{-1}(1, \lambda)=\left(\left[\begin{array}{cc}
I_{m} & H \\
0 & I_{m}
\end{array}\right] U(1, \lambda)\right)^{-1}=\left[\begin{array}{cc}
-S^{*}(1 ; \lambda) & {[V(S)]^{*}} \\
(\varphi)^{*}(1, \lambda) & -[V(\varphi)]^{*}
\end{array}\right] .
$$

Since

$$
U(x, \lambda) U^{-1}(x, \lambda)=I_{2 m}
$$

we have

$$
V(\varphi)[V(S)]^{*}=V(S)[V(\varphi)]^{*},
$$

i.e., $\mathcal{M}(\lambda)=V(\varphi)^{-1} V(S)$ is real symmetric for all $\lambda \in \mathbb{R}$. $\square$

Definition 2.1. We call $L_{m}(h, H, Q)$ a real symmetric problem if $h, H$ are real symmetric matrices and $Q(x)$ is a real symmetric matrix-valued function.

Corollary 2.5. Let $L_{m}(h, H, Q)$ and $L(\tilde{h}, \tilde{H}, \tilde{Q})$ be two real symmetric problems. Suppose that $\Delta_{i j}(\lambda)=\tilde{\Delta}_{i j}(\lambda)$ for $(i, j)=(0,0)$ or $1 \leq i \leq j \leq m$, then $h=\tilde{h}, h=\tilde{H}$ and $Q=\widetilde{Q}$.

Proof. For $\lambda \in \mathbb{R}$. both $\mathcal{M}(\lambda)$ and $\tilde{\mathcal{M}}(\lambda)$ are real symmetric. Moreover,

$$
\begin{aligned}
\mathcal{M}_{j i}(\lambda) & =\frac{\Delta_{00}(\lambda)-\Delta i j(\lambda)}{\Delta_{00}(\lambda)} \\
& =\frac{\tilde{\Delta}_{00}(\lambda)-\tilde{\Delta} i j(\lambda)}{\tilde{\Delta}_{00}(\lambda)} \\
& =\tilde{\mathcal{M}}_{j i}(\lambda), \text { for } 1 \leq i \leq j \leq m .
\end{aligned}
$$

Hence, $\mathcal{M}_{i j}(\lambda)=\tilde{\mathcal{M}}_{i j}(\lambda)$ for $\lambda \in \mathbb{R}$ and $1 \leq i, j \leq m$. This leads to $\Delta_{i j}(\lambda)=\tilde{\Delta}_{i j}(\lambda)$ for $\lambda \in \mathbb{R}$. We conclude that $\Delta_{i j}(\lambda)=\tilde{\Delta}_{i j}(\lambda)$ and $\mathcal{M}_{i j}(\lambda)=\tilde{\mathcal{M}}_{i j}(\lambda)$ for $\lambda \in \mathbb{C}$. This completes the proof. $\square$

From now on, we let $L_{m}(Q, h, H)$ be a real symmetric problem. We would like to know that how many spectral data can determine the problem $L_{m}(Q, h, H)$ if we require all spectral data come from real symmetric problems. Denote

$$
\begin{aligned}
\Gamma_{i j} & =\left[e_{1}, \ldots, \stackrel{(\text { ith-column })}{0}, \ldots, \stackrel{\text { (jth-column })}{0}, \ldots, e_{m}\right], \\
\Gamma^{i j} & =\left[0, \ldots, \stackrel{(\text { ith-column })}{e_{i}}, \ldots,{ }^{(\text {ith-column })} e_{j}, \ldots, 0\right],
\end{aligned}
$$

where $e_{i}=(0,0, \ldots, 0, \stackrel{\text { (ith-coordiante) }}{1}, 0, \ldots, 0)^{t}$. Hence, $\Gamma_{i j}+\Gamma^{i j}=I_{m}$. Let $\Theta_{i j}(\lambda)$ be the characteristic function of the self-adjoint problem

$$
\gamma^{\prime \prime}+\left(\lambda I_{m}-Q(x)\right) y=0, \quad 0<x<\pi
$$


associated with some boundary conditions

$$
\left\{\begin{array}{l}
\Gamma_{i j} \gamma^{\prime}(0, \lambda)-\left(\Gamma_{i j} h+\Gamma^{i j}\right) y(0, \lambda)=0, \\
\gamma^{\prime}(\pi, \lambda)+H \gamma(\pi, \lambda)=0,
\end{array}\right.
$$

then

$$
\Theta_{i j}(\lambda)=\operatorname{det}\left[V\left(\varphi_{1}\right), \ldots, \quad \stackrel{(\text { ith-column })}{V\left(S_{j}\right)}, \ldots, \quad \stackrel{(\text { ith-column })}{V\left(S_{i}\right)}, \ldots, V\left(\varphi_{m}\right)\right]
$$

where $V\left(L_{j}\right)$ denotes the $j$ th column of $(V(L))$ for a $m \times m$ matrix $L$. Similarly, we denote $\Omega_{i j}(\lambda)$ the characteristic function of the real symmetric problem $L_{m}\left(Q, h+\frac{1}{2}(B(i, j)+B(j, i)), H\right)$ for $1 \leq i, j \leq m$, then

$$
\begin{aligned}
& \Omega_{i j}(\lambda)=\operatorname{det}\left[\begin{array}{cc}
\text { (ith-column) } & \text { (jth-column) } \\
V\left(\varphi_{1}\right), \ldots, V\left(\varphi_{i}\right)+\frac{1}{2} V\left(S_{j}\right), \ldots, V\left(\varphi_{j}\right)+\frac{1}{2} V\left(S_{i}\right), \ldots, V\left(\varphi_{m}\right)
\end{array}\right] \\
& =\operatorname{det}\left[V\left(\varphi_{1}\right), \ldots, V\left(\varphi_{i}\right), \ldots, V\left(\varphi_{m}\right)\right]
\end{aligned}
$$

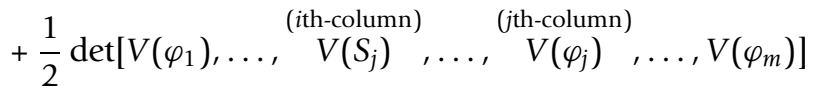

$$
\begin{aligned}
& +\frac{1}{2} \operatorname{det}\left[V\left(\varphi_{1}\right), \ldots, \quad \stackrel{(\text { ith-column })}{V\left(\varphi_{i}\right)}, \ldots, \quad{ }^{\text {(ith-column })}, \quad V\left(S_{i}\right), \ldots, V\left(\varphi_{m}\right)\right] \\
& +\operatorname{det}\left[V\left(\varphi_{1}\right), \ldots, \quad V\left(S_{j}\right), \ldots, \quad V\left(S_{i}\right), \ldots, V\left(\varphi_{m}\right)\right]
\end{aligned}
$$

for $1 \leq i, j \leq m$. For simplicity, we write

$$
\Omega_{00}(\lambda)=\operatorname{det}\left[V\left(\varphi_{1}\right), \ldots, V\left(\varphi_{j}\right), \ldots, V\left(\varphi_{m}\right)\right] .
$$

Now, we are going to focus on self-adjoint problems. For a self-adjoint problem $L_{m}$ $(Q, h, H)$ all its eigenvalues are real and the geometric multiplicity of an eigenvalue is equal to its algebraic multiplicity. Moreover, if we denote $\left\{\left(\lambda_{i}, m_{i}\right)\right\}_{i=1, \infty}$ the spectral data of $L_{m}(Q, h, H)$ where $m_{i}$ is the multiplicity of the eigenvalue $\lambda_{i}$ of $L_{m}(Q, h, H)$ then the characteristic function of $L_{m}(Q, h, H)$ is

$$
\Delta(\lambda)=C \Pi_{i=1}^{\infty}\left(1-\frac{\lambda}{\lambda_{i}}\right)^{m_{i}}
$$

where $C$ is determined by $\left\{\left(\lambda_{i}, m_{i}\right)\right\}_{i}=1, \infty$. This means that the spectral data determined the corresponding characteristic function.

Theorem 2.6. Assuming that $L_{m}(Q, h, H)$ and $L_{m}(\tilde{Q}, \tilde{h}, \tilde{H})$ are two real symmetric problems. If the conditions

(1) $\Omega_{i j}(\lambda)=\widetilde{\Omega}_{i j}(\lambda)$ for $(i, j)=(0,0)$ or $1 \leq i \leq j \leq m$,

(2) $\Theta_{i j}(\lambda)=\widetilde{\Theta}_{i j}(\lambda)$ for $1 \leq i<j \leq m$.,

are satisfied, then $h=\tilde{h}, H=\widetilde{H}$ and $Q(x)=\widetilde{Q}(x)$ a.e on $[0,1]$. 
Proof. Note that for any problem $L_{m}(Q, h, H)$ we have

$$
\begin{aligned}
\Delta_{i j}(\lambda) & =\operatorname{det}\left[V\left(\varphi_{1}\right), \ldots, \quad V\left(\varphi_{i}\right), \ldots, V\left(\varphi_{j}\right)+V\left(S_{i}\right), \ldots, V\left(\varphi_{m}\right)\right] \\
& =\operatorname{det}\left[V\left(\varphi_{1}\right), \ldots, V\left(\varphi_{j}\right), \ldots, V\left(\varphi_{m}\right)\right] \\
& +\operatorname{det}\left[V\left(\varphi_{1}\right), \ldots, \quad V\left(\varphi_{i}\right), \ldots, \quad V\left(S_{i}\right), \ldots, V\left(\varphi_{m}\right)\right] \\
& =\Delta_{00}(\lambda)+\operatorname{det}\left[V\left(\varphi_{1}\right), \ldots, \quad V\left(\varphi_{i}\right), \ldots, \quad V\left(S_{i}\right), \ldots, V\left(\varphi_{m}\right)\right] \\
& =\Delta_{00}(\lambda)-\Delta_{00}(\lambda) M_{j i}(\lambda) .
\end{aligned}
$$

Similarly,

$$
\tilde{\Delta}_{i j}(\lambda)=\tilde{\Delta}_{00}(\lambda)-\tilde{\Delta}_{00}(\lambda) \tilde{M}_{j i}(\lambda) .
$$

Moreover, by the assumptions and Lemma 2.4, we have $M_{i j}(\lambda)=M_{j i}(\lambda)$ Hence,

(1) $\Delta_{i j}(\lambda)=\Delta_{j i}(\lambda)$ and $\tilde{\Delta}_{i j}(\lambda)=\tilde{\Delta}_{j i}(\lambda)$ for $1 \leq i \leq j \leq m$,

(2) $\Delta_{i i}(\lambda)=\Omega_{i i}(\lambda)=\widetilde{\Omega}_{i i}(\lambda)=\widetilde{\Delta}_{i i}(\lambda)$ for $i=0,1, \ldots, m$,

(3) $\Delta_{i j}(\lambda)=\Omega_{i j}(\lambda)-\Theta_{i j}(\lambda)=\widetilde{\Omega}_{i j}(\lambda)-\widetilde{\Theta}_{i j}(\lambda)=\widetilde{\Delta}_{i j}(\lambda)$ for $1 \leq i<j \leq m$.

This implies $L_{m}(Q, h, H)=L_{m}(\tilde{Q}, \tilde{h}, \tilde{H})$.

The authors want to emphasis that for $n=1$, the result is classical; for $n=2$, Theorem 2.6 leads to Theorem 1.1. Shen also shows by providing an example that 5 minimal number of spectral sets can determine the potential matrix uniquely (see [18]).

The readers may think that if all $Q, h$ and $H$ are diagonals then $L_{m}(Q, h, H)$ is an uncoupled system. Hence, everything for the operator $L_{m}(Q, h, H)$ can be obtained by applying inverse spectral theory for scalar Sturm-Liouville equation. Unfortunately, it is not true. We say $L_{m}(Q, h, H)$ diagonal if all $Q, h$ and $H$ are diagonals.

Corollary 2.7. Suppose $L_{m}(Q, h, H)$ and $L_{m}(\widetilde{Q}, \tilde{h}, \widetilde{H})$ are both diagonals. If $\Delta_{k k}(\lambda)=\widetilde{\Delta}_{k k}(\lambda)$ for $k=0,1, \ldots, m$, then $Q=\widetilde{Q}, h=\tilde{h}$ and $H=\widetilde{H}$.

Proof. Since $L_{m}(Q, h, H)$ and $L_{m}(\widetilde{Q}, \tilde{h}, \widetilde{H})$ are both diagonals, we know

$$
\mathcal{M}(\lambda)=\frac{-\operatorname{Adj}\left(\varphi^{\prime}(\pi, \lambda)+H \varphi(\pi, \lambda)\right)}{\operatorname{det}\left(\varphi^{\prime}(\pi, \lambda)+H \varphi(\pi, \lambda)\right)}\left(S^{\prime}(\pi, \lambda)+H S(\pi, \lambda)\right)
$$

is diagonal and so is $\widetilde{\mathcal{M}}(\lambda)$. Hence,

$$
\mathcal{M}_{i j}(\lambda)=0 \text { for } i \neq j, 1 \leq i, j \leq m .
$$

Moreover,

$$
\begin{aligned}
\mathcal{M}_{k k}(\lambda) & =\frac{-1}{\Delta_{00}(\lambda)}\left(\varphi_{1}^{\prime}(\pi, \lambda)+H_{1} \varphi_{1}(\pi, \lambda) \cdots\left(S_{k}^{\prime}(\pi, \lambda)+H_{k} S_{k}(\pi, \lambda)\right) \cdots\right. \\
& =\frac{-1}{\Delta_{00}(\lambda)}\left(\Delta_{k k}(\lambda)-\Delta_{00}(\lambda)\right) \\
& =\frac{\Delta_{00}(\lambda)-\Delta_{k k}(\lambda)}{\Delta_{00}(\lambda)} \\
& =\frac{\widetilde{\Delta}_{00}(\lambda)-\widetilde{\Delta}_{k k}(\lambda)}{\widetilde{\Delta}_{00}(\lambda)} \\
& =\widetilde{\mathcal{M}}_{k k}(\lambda) .
\end{aligned}
$$


for $k=1,2, \ldots, m$. This implies. $\mathcal{M}(\lambda)=\widetilde{\mathcal{M}}(\lambda)$. Applying Theorem 2.1 again, we have $Q=\widetilde{Q}, h=\tilde{h}$ and $H=\widetilde{H}$.

\section{Footnote}

This work was partially supported by the National Science Council, Taiwan, ROC.

\section{Author details}

'Department of Mathematics, Tamkang University, No.151, Yingzhuan Rd., Danshui Dist., New Taipei City 25137, Taiwan, PR China ${ }^{2}$ Department of Electronic Engineering, China University of Science and Technology, No.245, Academia Rd., Sec. 3, Nangang District, Taipei City 115, Taiwan, PR China

\section{Authors' contributions}

Both authors contributed to each part of this work equally and read and approved the final version of the manuscript.

\section{Competing interests}

The authors declare that they have no competing interests.

Received: 28 April 2011 Accepted: 26 October 2011 Published: 26 October 2011

\section{References}

1. Borg, G: Eine Umkehrung der Sturm-Liouvilleschen Eigenwertaufgabe Bestimmung der Differentialgleichung durch die Eigenwerte. Acta Math. 78, 1-96 (1945)

2. Gesztesy, F, Simon, B: On the determination of a potential from three spectra. In: Differential Operators and Spectral Theory. In Am Math Soc Transl Ser 2, vol. 189, pp. 85-92.American Mathematical Society, Providence, RI (1999)

3. Hochstadt, H: The inverse Sturm-Liouville problem. Commun Pure Appl Math. 26, 75129 (1973)

4. Hochstadt, H, Lieberman, B: An inverse Sturm-Liouville problem with mixed given data. SIAM J Appl Math. 34, 67680 (1978)

5. Kren, MG: Solution of the inverse Sturm-Liouville problem. Dokl Akad Nauk SSSR. 76, 214 (1951)

6. Levitan, BM: Inverse Sturm-Liouville Problems. VNM, Utrecht (1987)

7. Levitan, BM, Gasymov, MG: Determination of a differential equation by two of its spectra. Russ Math Surv. 19, 163 (1964). doi:10.1070/RM1964v019n03ABEH001151

8. Marchenko, VA: Sturm-Liouville Operators and Applications. Birkhauser, Basel. (1986)

9. Yurko, VA: Method of spectral mappings in the inverse problem theory. Inverse and III-Posed Problems Series. (2002)

10. Andersson, E: On the M-function and Borg-Marchenko theorems for vector-valued Sturm-Liouville equations. J Math Phys.44(12), 6077-6100

11. Carlson, R: An inverse problem for the matrix Schrödinger equation. J Math Anal Appl. 267, 564-575 (2002). doi:10.1006/jmaa.2001.7792

12. Chern, H-H, Shen, C-L: On the n-dimensional Ambarzumyan's theorem. Inverse Probl.13(1), 15-18

13. Clarka, S, Gesztesy, F, Holdenc, H, Levitand, BM: Borg-Type theorems for matrix-valued Schrödinger operators. J Differ Equ. 167(1), 181-210 (2000). doi:10.1006/jdeq.1999.3758

14. Gesztesy, F, Kiselev, A, Makarov, K-A: Uniqueness results for matrix-valued Schrödinger, Jacobi, and Dirac-Type operators. Math Nachr. 239-240(1), 103-145 (2002). doi:10.1002/1522-2616(200206)239:13.0.CO;2-F

15. Jodeit, M, Levitan, BM: The isospectrality problem for the classical Sturm-Liouville equation. Adv Differ Equ. 22, 297-318 (1997)

16. Jodeit, M, Levitan, BM: Isospectral vector-valued Sturm-Liouville problems. Lett Math Phys. 43, 117-122 (1998). doi:10.1023/A:1007498010532

17. Shen, C-L: Some eigenvalue problems for the vectorial Hill's equation. Inverse Probl. 16(3), 749-783 (2000). doi:10.1088/ $0266-5611 / 16 / 3 / 313$

18. Shen, C-L: Some inverse spectral problems for vectorial Sturm-Liouville equations. Inverse Probl. 17(5), 1253-1294 (2001). doi:10.1088/0266-5611/17/5/303

19. Shieh, C-T: Isospectral sets and inverse problems for vector-valued Sturm-Liouville equations. Inverse Probl. 23(6), 2457-2468 (2007). doi:10.1088/0266-5611/23/6/011

20. Yurko, VA: Inverse problems for the matrix Sturm-Liouville equation on a finite intervl. Inverse Probl. 22, 1139-1149 (2006). doi:10.1088/0266-5611/22/4/002

doi:10.1186/1687-2770-2011-40

Cite this article as: Chang and Shieh: Uniqueness of the potential function for the vectorial Sturm-Liouville equation on a finite interval. Boundary Value Problems 2011 2011:40. 